\title{
ERGODIC THEOREMS FOR THE ASYMMETRIC SIMPLE EXCLUSION PROCESS $\left({ }^{1}\right)$
}

\author{
BY
}

\author{
THOMAS M. LIGGETT
}

\begin{abstract}
Consider the infinite particle system on the integers with the simple exclusion interaction and one-particle motion determined by $p(x, x+1)=$ $p$ and $p(x, x-1)=q$ for $x \in Z$, where $p+q=1$ and $p>q$. If $\mu$ is the initial distribution of the system, let $\mu_{t}$ be the distribution at time $t$. The main results determine the limiting behavior of $\mu_{t}$ as $t \rightarrow \infty$ for simple choices of $\mu$. For example, it is shown that if $\mu$ is the pointmass on the configuration in which all sites to the left of the origin are occupied, while those to the right are vacant, then the system converges as $t \rightarrow \infty$ to the product measure on $\{0,1\}^{Z}$ with density $1 / 2$. For the proof, an auxiliary process is introduced which is of interest in its own right. It is a process on the positive integers in which particles move according to the simple exclusion process, but with the additional feature that there can be creation and destruction of particles at one. Ergodic theorems are proved for this process also.
\end{abstract}

1. Introduction. Let $p(x, y)$ be the transition function for a Markov chain on the countable set $S$. The infinite particle system on $S$ with the simple exclusion interaction and one-particle motion determined by $p$ was introduced by Spitzer in [8], and can be described in the following way. Particles are distributed initially on $S$ in such a way that there is at most one particle per site. Each particle waits an exponential time with parameter one, and then attempts a transition to another site in $S$ chosen according to the probabilities $p(x, y)$. It makes the transition if that site is vacant, while if it is occupied, the particle remains where it was. As a result of this exclusion interaction, some condition on $p(x, y)$ is needed to guarantee that a process behaving according to this description exists. It was proved in [6] that a sufficient condition for the existence of such a process is that $\sup _{y} \Sigma_{x} p(x, y)<\infty$. The resulting system is then a strong Markov process $\eta_{t}$ with state space $X=\{0,1\}^{S}$ whose infinitesimal generator is the closure in $C(X)$ of the operator $\Omega$ defined for functions $f$ which depend on only finitely many coordinates by

Received by the editors August 31, 1974.

AMS (MOS) subject classifications (1970). Primary 60K35; Secondary 47A35.

Key words and phrases. Infinite particle systems, simple exclusion process.

(1) Research supported in part by NSF Grant No. GP-33431X, and in part by an Alfred P. Sloan Foundation research fellowship. 


$$
\Omega f(\eta)=\sum_{\substack{\eta(x)=1 \\ \eta(y)=0}} p(x, y)\left[f\left(\eta_{x y}\right)-f(\eta)\right],
$$

where $\eta_{x y}$ denotes the configuration obtained from $\eta$ by interchanging the $x$ and $y$ coordinates. Let $S(t)$ be the semigroup on $C(X)$ which corresponds to this generator. Recall that if $\mu$ is the initial distribution of the process, then $\mu S(t)$ is the distribution of the process at time $t$.

There are two basic problems which arise in the ergodic theory of the process $\eta_{t}$. The first is to describe the set of all invariant (probability) measures for the system: $I=\{\mu$ on $X: \mu S(t)=\mu$ for all $t>0\}$, while the second is to determine the domain of attraction of each extreme invariant measure. This means that for a given $\mu \in I_{e}$, one wants to find all probability measures $\nu$ for which $\nu S(t) \rightarrow \mu$ weakly as $t \rightarrow \infty$. These questions were answered completely in [3], [4] and [9] under the assumption that $p(x, y)=p(y, x)$ for all $x, y \in S$. The property which makes this symmetric case tractable is contained in a theorem due to Spitzer (see [3, Theorem 1.1]) which permits the reduction of many problems involving the infinite particle system to ones involving the corresponding finite system. Analytically, the effect of this is that the $n$-point probabilities $[\nu S(t)]\{\eta$ : $\eta\left(x_{1}\right)=1, \ldots, \eta\left(x_{n}\right)=1$ \} of $\nu S(t)$ can be expressed in terms of the $n$-point probabilities of $\nu$ for the same $n$. If $p$ is not symmetric, Spitzer's theorem fails and, in fact, the $n$-point probabilities of $\nu S(t)$ depend on the full structure of the measure $\nu$ in a very complex way. This makes the analysis much more difficult, and there is little hope that anything resembling the complete results available in the symmetric case can be obtained in general. In fact, it will be seen in this paper that the difficulty is not merely the absence of the analytical tools provided in the symmetric case by Spitzer's theorem, but also the fact that the limiting behavior of the system is more complicated in the asymmetric case. One asymmetric case which has been treated is that in which $p$ is positive recurrent and reversible [5]. This case is not at all typical, however, since one finds that there are no measures in $I$ which concentrate on $\left\{\eta \in X: \Sigma_{x} \eta(x)=\infty, \Sigma_{x}[1-\eta(x)]\right.$ $=\infty$.

In this paper, we consider the case in which $S=Z$ (the integers) and

$$
p(x, x+1)=p \text { and } p(x, x-1)=q
$$

for all $x \in Z$, where $p+q=1$. Some of our techniques of proof will probably not generalize significantly beyond this case, but some of them will, and our results should indicate to some extent what may be expected to hold in greater generality. We will assume that $p \neq 1 / 2$, since otherwise $p(x, y)$ is symmetric and the ergodic theory of $\eta_{t}$ is covered by the results of [9]. Furthermore, there is an obvious translation of results for $p>1 / 2$ into corresponding results for $p<1 / 2$, so we will assume throughout that $1 / 2<p \leqslant 1$. 
We begin by stating what is known about $I$, since we have little new to add to its description. For $\rho \in[0,1]$, let $\nu_{\rho}$ be the product measure on $X$ with $\nu_{\rho}\{\eta: \eta(x)=1\}=\rho$ for all $x \in Z$. In [1], Holley proved that $\nu_{\rho} \in I$ for each $\rho$. (In fact, this is true whenever $p(x, y)$ is doubly stochastic, as was conjectured by Spitzer in [8] and can be easily proved using the results of [6].) These are the only extreme invariant measures if $p=1 / 2$, as was proved in [9]. In our case, however, there is another class of measures in I. If $p=1$ and $n \in Z$, let $v_{n}$ be the pointmass on the configuration in which $\eta(x)=1$ for $x \geqslant n$ and $\eta(x)=0$ for $x<n$. This is clearly invariant for the process, since, with this initial configuration, no transitions are possible. If $1 / 2<p<1$, there is a corresponding collection of invariant measures which can be described in the following way. Let $r=p / q$ and for $0<\rho<\infty$ let $\tilde{\nu}_{\rho}$ be the product measure on $X$ with $\tilde{\nu}_{\rho}\{\eta: \eta(x)=1\}=$ $\rho r^{x} /\left(1+\rho r^{x}\right)$. The proof of part (a) of [5, Theorem 3.1] is independent of the assumption of positive recurrence of $p(x, y)$, so it follows from that result that $\tilde{\nu}_{\rho} \in I$ for each $\rho$ since $r^{x} p(x, y)=r^{y} p(y, x)$. Let $A_{n}=\left\{\eta \in X: \Sigma_{x<n} \eta(x)=\right.$ $\left.\Sigma_{x>n}[1-\eta(x)]<\infty\right\}$ and $A=\left\{\eta \in X: \Sigma_{x<0} \eta(x)<\infty\right.$ and $\left.\Sigma_{x>0}[1-\eta(x)]<\infty\right\}$. Then $A=\bigcup_{n=-\infty}^{\infty} A_{n}$, and $\tilde{\nu}_{\rho}(A)=1$ for each $\rho$ since $r>1$. Since $A$ is countable, $\eta_{t}$ is simply a Markov chain on $A$, and it is easy to check that $\left\{A_{n}:-\infty<\right.$ $n<\infty\}$ are the closed irreducible classes for the chain. Therefore the restriction of $\tilde{\nu}_{\rho}$ to $A_{n}$ is invariant for $\eta_{t}$, and since $\tilde{\nu}_{\rho}\left(A_{n}\right)>0$ for each $n$ and $\rho$, the chain is positive recurrent on each $A_{n}$ and has stationary distribution $\nu_{n}=\widetilde{\nu}_{\rho}\left(\cdot \mid A_{n}\right)$, which is therefore independent of $\rho$. Presumably, the only extreme invariant measures for the process are $\left\{v_{\rho}: 0 \leqslant \rho \leqslant 1\right\}$ and $\left\{\nu_{n}:-\infty<n<\infty\right\}$, but the proof of this remains an open problem. The one result along these lines which is available is that the only invariant measures which are translation invariant are the exchangeable measures on $X$. A proof of this statement can be constructed using the methods developed by Holley in [2]. Added in proof: The above conjecture is correct, and will be proved in a forthcoming paper by the present author.

We turn now to a description of our results on the convergence problem.

The class of initial distributions which concentrate on $A$ can be treated immediately. If $\mu(A)=1$, and in particular if $\Sigma_{x<0} \mu\{\eta: \eta(x)=1\}<\infty$ and $\Sigma_{x>0} \mu\{\eta: \eta(x)=0\}$ $<\infty$, the ordinary convergence theorem for positive recurrent Markov chains yields

$$
\lim _{t \rightarrow \infty} \mu S(t)=\sum_{n=-\infty}^{\infty} \mu\left(A_{n}\right) \nu_{n} .
$$

Our main interest, however, is treating initial distributions $\mu$ which are product measures on $X$ for which $\lim _{x \rightarrow \infty} \mu\{\eta: \eta(x)=1\}$ and $\lim _{x \rightarrow-\infty} \mu\{\eta: \eta(x)=1\}$ both exist but are not necessarily equal. For such initial distributions, there are two components of the motion which would seem to determine the limiting behavior of the process as $t \rightarrow \infty$. For example, suppose $\mu$ has low density on the left and high density on the right. Since $p>q$, one might think that the whole 
system would tend to move toward the right, and that therefore one would tend to observe the lower density situation as $t \rightarrow \infty$. This would in fact happen if there were no interaction. On the other hand, since particles will interfere with each other's motion more at high density than at low density, the particles on the left will initially move to the right more rapidly than will those on the right, and will meet increasing interference from those on the right. Therefore the density of the particles on the left will increase, and one might expect to observe the high density situation as $t \rightarrow \infty$. Our main theorem describes conditions under which one or the other or both of these mechanisms dominate the evolution of the process.

Theorem 1.3. Assume that $\mu$ is a product measure on $X$ and that the following limits exist:

$$
\lambda=\lim _{x \rightarrow-\infty} \mu\{\eta: \eta(x)=1\}, \quad \rho=\lim _{x \rightarrow+\infty} \mu\{\eta: \eta(x)=1\} .
$$

(a) If $\lambda \geqslant 1 / 2$ and $\rho \leqslant 1 / 2$, then $\lim _{t \rightarrow \infty} \mu S(t)=\nu_{1 / 2}$.

(b) If $\rho \geqslant 1 / 2$ and $\lambda+\rho>1$, then $\lim _{t \rightarrow \infty} \mu S(t)=\nu_{\rho}$.

(c) If $\lambda \leqslant 1 / 2$ and $\lambda+\rho<1$, then $\lim _{t \rightarrow \infty} \mu S(t)=\nu_{\lambda}$.

It is interesting to note that the effect of the interaction is much more pronounced in this case than in the symmetric case. For purposes of comparison, it should be mentioned that if $p=1 / 2$, Theorem 4 of [9] implies that under the above conditions, $\lim _{t \rightarrow \infty} \mu S(t)=\nu_{(\lambda+\rho) / 2}$ for any choice of $\lambda$ and $\rho$.

The above theorem omits the case

$$
\lambda+\rho=1 \text { and } \lambda<1 / 2
$$

in which the situation is much more delicate. In fact, in this case, (1.4) is not sufficient to guarantee convergence at all. It will be shown as a consequence of Theorem 1.3 that if $\lambda$ and $\rho$ satisfy (1.5), there exists a product measure $\mu$ satisfying (1.4) for which $\mu S(t)$ has both $\nu_{\lambda}$ and $\nu_{\rho}$ as weak limit points as $t \rightarrow \infty$. However we do make the following conjecture in case the convergence in (1.4) is sufficiently rapid.

Conjecture 1.6. Assume that $\mu$ is a product measure on $X$ which satisfies $\Sigma_{x<0}|\mu\{\eta: \eta(x)=1\}-\lambda|<\infty$ and $\Sigma_{x>0}|\mu\{\eta: \eta(x)=1\}-\rho|<\infty$. If $\lambda+\rho=1$ and $0<\lambda<1 / 2$, then $\lim _{t \rightarrow \infty} \mu S(t)=1 / 2 v_{\lambda}+1 / 2 v_{\rho}$.

REMARK. It follows from (1.2) that this is not true if $\lambda=0$ and $\rho=1$.

In the course of the proof of Theorem 1.3, an auxiliary process which is of interest in its own right is introduced and studied. It is a Markov process on $X_{+}=\{0,1\}^{Z_{+}}$where $Z_{+}=\{1,2, \ldots\}$ which can be described as the simple exclusion process on $Z_{+}$with the additional feature that there can be creation and destruction of particles at one. The creation and destruction rates at one 
depend on only one parameter $\lambda \in[0,1]$, and are chosen in such a way that they can be viewed as arising from a fictitious state 0 with the property that $\eta_{t}(0)=1$ with probability $\lambda$ and $\eta_{t}(0)$ is independent of the process $\left\{\eta_{t}(x), x \in Z_{+}\right\}$for all $t \geqslant 0$. Thus the creation and destruction rates at one are $p \lambda$ and $q(1-\lambda)$ respectively, and the generator of the process, when restricted to functions $f$ which depend on finitely many coordinates, is given by

$$
\begin{aligned}
\Omega_{\lambda} f(\eta)= & \sum_{x=1}^{\infty}\{p \eta(x)[1-\eta(x+1)]+q \eta(x+1)[1-\eta(x)]\}\left[f\left(\eta_{x, x+1}\right)-f(\eta)\right] \\
& +\{p \lambda[1-\eta(1)]+q(1-\lambda) \eta(1)\}\left[f\left(\eta_{1}\right)-f(\eta)\right]
\end{aligned}
$$

where $\eta_{1}(x)=\eta(x)$ for $x>1$ and $\dot{\eta}_{1}(1)=1-\eta(1)$. Let $S_{\lambda}(t)$ be the semigroup corresponding to this generator. We will refer to the process as the $\lambda$-process.

It is easy to check that $\nu_{\lambda}$ is invariant for this process. In fact if $p=1 / 2$, this is the only invariant measure and $\mu S_{\lambda}(t) \rightarrow \nu_{\lambda}$ for every initial distribution $\mu$. However, in our case in which $p>1 / 2$, there are in general other invariant measures as described in the following theorem. We will say that a probability measure $\mu$ on $X_{+}$behaves like $\nu_{\rho}$ at $\infty$ if

$$
\lim _{x \rightarrow \infty} \mu\left\{\eta: \eta\left(x_{1}+x\right)=1, \ldots, \eta\left(x_{n}+x\right)=1\right\}=\rho^{n}
$$

for all distinct choices $x_{1}, \ldots, x_{n} \in Z$ and all $n \geqslant 1$.

THEOREM 1.7. There exists a collection $\mu(\lambda, \rho)$ of probability measures on $X_{+}$defined for $1 / 2 \leqslant \rho \leqslant 1$ if $1 / 2 \leqslant \lambda \leqslant 1$ and for $\rho=\lambda$ and $\rho>1-\lambda$ if $0 \leqslant$ $\lambda \leqslant 1 / 2$ with the following properties:

(a) $\mu(\lambda, \rho)$ is invariant for the $\lambda$-process for each $\rho$.

(b) $\mu(\lambda, \rho)$ behaves like $\nu_{\rho}$ at $\infty$.

(c) $\mu(\lambda, \lambda)=v_{\lambda}$.

(d) $\mu(\lambda, \rho)$ is jointly weakly continuous in $\lambda$ and $\rho$.

(e) If $0<\lambda<1 / 2$, then $v_{\lambda}=\lim _{\rho \downarrow 1-\lambda} \mu(\lambda, \rho)$.

The convergence results for the $\lambda$-process which are used in the proof of Theorem 1.3 are given next.

THEOREM 1.8. Assume that $\mu$ is a product measure on $X_{+}$for which $\rho=$ $\lim _{x \rightarrow \infty} \mu\{\eta: \eta(x)=1\}$ exists.

(a) If $\lambda \geqslant 1 / 2$, then $\lim _{t \rightarrow \infty} \mu S_{\lambda}(t)=\mu(\lambda, \rho)$ if $\rho \geqslant 1 / 2$, and $\lim _{t \rightarrow \infty} \mu S_{\lambda}(t)=$ $\mu(\lambda, 1 / 2)$ if $\rho \leqslant 1 / 2$.

(b) If $\lambda \leqslant 1 / 2$, then $\lim _{t \rightarrow \infty} \mu S_{\lambda}(t)=\mu(\lambda, \rho)$ if $\rho>1-\lambda$, and $\lim _{t \rightarrow \infty} \mu S_{\lambda}(t)$ $=\nu_{\lambda}$ if $\rho \leqslant 1-\lambda$ If $\lambda=0, \rho=1$ and $p=1$, the additional assumption that $\Sigma_{x \geqslant 1} \mu\{\eta: \eta(x)=0\}=\infty$ is required. 
An interesting special case of this theorem is that in which the process begins with $\eta(x)=0$ for all $x \in Z_{+}$. Then we see that $\mu S_{\lambda}(t) \rightarrow \nu_{\lambda}$ if $\lambda \leqslant 1 / 2$, but $\mu S_{\lambda}(t)$ converges to a measure which behaves like $\nu_{1 / 2}$ at $\infty$ if $\lambda \geqslant 1 / 2$. Thus the mechanism of creation of particles at one appears not to be sufficiently strong if $p>1 / 2$ to raise the asymptotic density of particles above $1 / 2$.

In the proofs of these results, it will of ten be necessary to compare the distributions at time $t$ of two different processes which began with the same distribution, or of the same process begun with different distributions. The inequalities between probability measures which will be needed in this connection will be described and proved in $\S 2 . \S 3$ is devoted to the analysis of a process on $\{1$, $\ldots, n\}$ which has creation and destruction of particles at both 1 and $n$. This process is simply a Markov chain with a finite state space, and the aim here is to study its stationary distribution, and in particular the limiting behavior of the stationary distribution as $n \rightarrow \infty$. Theorems 1.7 and 1.8 will then be proved in $\S 4$ by comparing the $\lambda$-process with the finite system. Finally, Theorem 1.3 will be proved in $\S 5$ by comparison with the $\lambda$-process.

2. Comparisons and inequalities. Let $p(x, y)$ be a doubly stochastic transition function for a Markov chain on the countable set $S$, and let $\alpha(x)$ be a function on $S$ which satisfies $0 \leqslant \alpha(x) \leqslant 1$ for all $x$. For each subset $D$ of $S$, we will consider the Markov process on $X_{D}=\{0,1\}^{D}$ whose generator, when restricted to functions depending on finitely many coordinates, has the form

$$
\begin{aligned}
\Omega_{D} f(\eta)= & \sum_{x, y \in D} \eta(x)[1-\eta(y)] p(x, y)\left[f\left(\eta_{x, y}\right)-f(\eta)\right] \\
& +\sum_{\substack{x \in D \\
y \notin D}}\{\eta(x) p(x, y)[1-\alpha(y)]+[1-\eta(x)] p(y, x) \alpha(y)\}\left[f\left(\eta_{x}\right)-f(\eta)\right],
\end{aligned}
$$

where again $\eta_{x}(y)=\eta(y)$ for $y \neq x$ and $\eta_{x}(x)=1-\eta(x)$. Since $p$ is doubly stochastic, the existence results of [6] guarantee that there is a unique generator with this restriction. For this process, in addition to the motion on $D$ according to the simple exclusion interaction, there is spontaneous creation and destruction of particles at rates which depend on the function $\alpha$. Note that if $D=S$, this is the ordinary simple exclusion process on $S$, while the $\lambda$-process described in the introduction is obtained by taking $S=Z, D=Z_{+}$and $\alpha(0)=\lambda$. Let $S_{D}(t)$ denote the semigroup corresponding to this process. When dealing with a probability measure on $X_{D}$ which represents the distribution of the process at some time, we will adopt the convention that it is to be regarded as a probability measure on $X$ by letting it be the product measure on $X=X_{D} \times \Pi_{x \in D}\{0,1\}$ with $\mu\{\eta: \eta(x)=1\}=\alpha(x)$ for $x \notin D$. Functions on $X_{D}$ will be viewed as functions on $X$ in the standard way. 
Define a partial order on probability measures on $X$ in the following way. Let $M$ be the set of monotone continuous functions on $X$ :

$$
M=\{f \in C(X): \eta, \zeta \in X \text { and } \eta \leqslant \zeta \Rightarrow f(\eta) \leqslant f(\zeta)\}
$$

If $D \subset S, M_{D}$ will denote the set of $f \in M$ which depend only on coordinates in $D$. Say that $\mu \leqslant \nu$ if $\int f d \mu \leqslant \int f d \nu$ for all $f \in M$. An equivalent definition is that $\mu \leqslant \nu$ if there exists a probability measure on $\{(\eta, \zeta) \in X \times X: \eta \leqslant \zeta\}$ whose marginal distributions are $\mu$ and $\nu$ respectively. The equivalence of these definitions is a consequence of $[7$, Theorem 53, Chapter XI]. In developing the relationship between this partial order and the process with generator $\Omega_{D}$, we will need the following two standard semigroup results. The first is in fact valid for any bounded generator, while the second is a consequence of the Trotter theorem on convergence of a sequence of semigroups.

Proposition 2.1. Suppose that $D$ is a finite subset of $S$ and that $K$ is a closed convex subset of $C\left(X_{D}\right)$. If there is an $\epsilon>0$ so that $I+\epsilon \Omega_{D}: K \rightarrow K$, then $S_{D}(t): K \rightarrow K$ for all $t \geqslant 0$.

Proposition 2.2. If $D_{n} \subset S$ and $D_{n} \uparrow D$, then, for all $f \in C\left(X_{D}\right)$ and $t \geqslant 0, S_{D_{n}}(t) f \rightarrow S_{D}(t) f$.

The following is a consequence of $[5$, Theorem 2.1] in case $D=S$.

THEOREM 2.3. If $\mu \leqslant \nu$, then $\mu S_{D}(t) \leqslant \nu S_{D}(t)$ for all $t \geqslant 0$ and $D \subset S$.

Proof. By Proposition 2.2, it suffices to prove the theorem in case $D$ is finite. So assume that $D$ has $n$ elements. If $f \in M_{D}$ and $x, y \in D$, then the functions $\eta(x)[1-\eta(y)] f\left(\eta_{x y}\right)+[1-\eta(x)][1-\eta(y)] f(\eta), \eta(x) f\left(\eta_{x}\right)+[1-\eta(x)] f(\eta)$, and $[1-\eta(x)] f\left(\eta_{x}\right)+\eta(x) f(\eta)$ are also in $M_{D}$. Therefore $\Omega_{D} f+2 n f \in M_{D}$, and hence $S_{D}(t): M_{D} \rightarrow M_{D}$ for all $t \geqslant 0$ by Proposition 2.1. The required result then follows from the relation $\int f d\left[\mu S_{D}(t)\right]=\int S_{D}(t) f d \mu$.

As mentioned in the introduction, the fact that $p(x, y)$ is doubly stochastic implies that $\nu_{\rho}$ is invariant for $S(t)$ for $0 \leqslant \rho \leqslant 1$. For $D \subset S$, the role of $\nu_{\rho}$ is described by the following result. In this, as well as in other theorems of this section, versions can be obtained with inequalities reversed simply by interchanging the roles of 0 and 1 .

THEOREM 2.4. (a) If $\alpha_{1}(x) \leqslant \alpha_{2}(x)$ on $S \backslash D$ and $\mu \leqslant \nu$, then $\mu S_{D}^{1}(t) \leqslant$ $\nu S_{D}^{2}(t)$, where $S_{D}^{i}(t)$ is the semigroup corresponding to $\alpha_{i}$. (b) If $\alpha(x)=\rho$ on $S \backslash D$, then $\nu_{\rho}$ is invariant for $S_{D}(t)$. (c) If $\alpha(x) \geqslant \rho$ on $S \backslash D$, then $\nu_{\rho} S_{D}(t) \geqslant \nu_{\rho}$.

Proof. Again it suffices to consider the case in which $D$ is finite. If $\Omega_{D}^{i}$ is the generator of $S_{D}^{i}(t)$, then 


$$
\begin{aligned}
\Omega_{D}^{2} f(\eta)- & \Omega_{D}^{1} f(\eta) \\
& =\sum_{\substack{x \in D \\
y \in D}}\{[1-\eta(x)] p(y, x)-\eta(x) p(x, y)\}\left[\alpha_{2}(y)-\alpha_{1}(y)\right]\left[f\left(\eta_{x}\right)-f(\eta)\right] .
\end{aligned}
$$

If $f \in M_{D}$, then $\eta(x)\left[f\left(\eta_{x}\right)-f(\eta)\right] \leqslant 0$ and $[1-\eta(x)]\left[f\left(\eta_{x}\right)-f(\eta)\right] \geqslant 0$, so, for such $f$,

$$
\Omega_{D}^{2} f(\eta) \geqslant \Omega_{D}^{1} f(\eta)
$$

Let $K=\left\{\left(f_{1}, f_{2}\right) \in M_{D} \times M_{D}: 0 \leqslant f_{1} \leqslant f_{2}\right\}$. By the proof of Theorem 2.3, there is an $\epsilon>0$ so that $I+\epsilon \Omega_{D}^{1}: M_{D} \rightarrow M_{D}$ and $I+\epsilon \Omega_{D}^{2}: M_{D} \rightarrow M_{D}$. Since $\Omega_{D}^{1}$ and $\Omega_{D}^{2}$ are the generators for finite Markov chains, $\epsilon$ can be made smaller in such a way that in addition $I+\epsilon \Omega_{D}^{1}$ and $I+\epsilon \Omega_{D}^{2}$ map nonnegative functions to nonnegative functions. Then, by $(2.5),\left(f_{1}, f_{2}\right) \in K$ implies that $\left(f_{1}+\epsilon \Omega_{D}^{1} f_{1}\right.$, $\left.f_{2}+\epsilon \Omega_{D}^{2} f_{2}\right) \in K$. Therefore $\left(S_{D}^{1}(t), S_{D}^{2}(t)\right)$ maps $K$ into itself. In particular, it follows that $S_{D}^{1}(t) f \leqslant S_{D}^{2}(t) f$ for all $f \in M_{D}$, and therefore that $\mu S_{D}^{1}(t) \leqslant \mu S_{D}^{2}(t)$ for all $\mu$. Part (a) then follows from Theorem 2.3. In order to prove part (b), note that, for any function $f$ which depends only on the coordinates in $D$,

$$
\Omega_{D} f(\eta)-\Omega f(\eta)=\sum_{\substack{x \in D \\ y \notin D}}\{\eta(x) p(x, y)-[1-\eta(x)] p(y, x)\}[\eta(y)-\rho]\left[f\left(\eta_{x}\right)-f(\eta)\right] .
$$

The integral of the right side with respect to $\nu_{\rho}$ is zero, and therefore $\int \Omega_{D} f(\eta) d \nu_{\rho}$ $=0$ since $\nu_{\rho}$ is invariant for $S(t)$. Part (c) then follows from (a) and (b).

Corollary 2.6. If $\alpha(x) \geqslant \rho$ on $S \backslash D$, then $\mu \geqslant \nu_{\rho}$ implies that

$$
\mu S_{D}(t) \geqslant \nu_{\rho} S_{D}(t) \geqslant \nu_{\rho} .
$$

A stronger form of this result will be needed. We will say that $\mu>_{s} \nu_{\rho}$ if

$$
\int f g d \mu \geqslant 0
$$

whenever

$$
\begin{aligned}
& f \in M, \int f d v_{\rho}=0, g \geqslant 0 \text {, and } f \text { and } g \text { depend } \\
& \text { on finite disjoint sets of coordinates. }
\end{aligned}
$$

By taking $g \equiv 1$, it can be seen that $\mu>_{s} \nu_{\rho}$ implies that $\mu \geqslant \nu_{\rho}$, thus justifying the notation. In fact, $\mu>_{s} \nu_{\rho}$ can be viewed as a conditioned form of $\mu \geqslant \nu_{\rho}$. The following result says that it suffices in the above definition to consider only functions $f$ which depend on one coordinate.

Lemma 2.9. Suppose that $\mu$ satisfies $\int[\eta(u)-\rho] g(\eta) d \mu \geqslant 0$ whenever $g$ is 
a nonnegative function which depends on finitely many coordinates other than $\eta(u)$. Then $\mu>_{s} \nu_{\rho}$.

Proof. Assume that $0<\rho<1$, since otherwise the result is immediate. The proof of (2.7) is by induction on the number of coordinates on which $f$ depends. If $f$ depends on only one coordinate $\eta(u)$, then $f$ is a nonnegative constant multiple of $[\eta(u)-\rho]$, so $(2.7)$ is true by assumption. Now let $f$ and $g$ be as in (2.8), and let $\eta(u)$ be one of the coordinates on which $f$ depends. Write $f(\eta)=[1-\eta(u)] f_{0}(\eta)+\eta(u) f_{1}(\eta)$, where $f_{0}$ and $f_{1}$ do not depend on the coordinate $\eta(u)$, and therefore depend on fewer coordinates than does $f$. Since $f \in$ $M$, it follows that $f_{0}, f_{1} \in M$ and $f_{0}(\eta) \leqslant f_{1}(\eta)$ for all $\eta$. Since $\int f d \nu_{\rho}=0$, it follows that

$$
(1-\rho) \int f_{0} d \nu_{\rho}+\rho \int f_{1} d \nu_{\rho}=0
$$

and so $\int f_{0} d \nu_{\rho} \leqslant 0 \leqslant \int f_{1} d \nu_{\rho}$. By the induction assumption,

$$
\int[1-\eta(u)] f_{0}(\eta) g(\eta) d \mu \geqslant\left[\int f_{0} d \nu_{\rho}\right] \int[1-\eta(u)] g(\eta) d \mu,
$$

and

$$
\int \eta(u) f_{1}(\eta) g(\eta) d \mu \geqslant\left[\int f_{1} d \nu_{\rho}\right] \int \eta(u) g(\eta) d \mu
$$

Therefore, by (2.10),

$$
\begin{aligned}
\int f g d \mu & \geqslant(1-\rho)^{-1}\left[\int f_{1} d v_{\rho}\right]\left[(1-\rho) \int \eta(u) g(\eta) d \mu-\rho \int[1-\eta(u)] g(\eta) d \mu\right] \\
& =(1-\rho)^{-1}\left[\int f_{1} d \nu_{\rho}\right]\left[\int[\eta(u)-\rho] g(\eta) d \mu\right] \geqslant 0,
\end{aligned}
$$

thus completing the induction step.

THEOREM 2.11. Suppose that $D$ is a finite subset of $S$. For fixed $\rho \in[0,1]$, let $L$ be the (closed, convex) set of all functions of the form $g(\eta)$ $+\Sigma_{x \in D}[\eta(x)-\rho] g_{x}(\eta)$, where $g$ and $g_{x}$ are nonnegative functions which depend only on the coordinates $\eta(y)$ for $y \in D$ and $y \in D \backslash\{x\}$ respectively. If $\alpha(x) \geqslant \rho$ on $S \bigvee$, then there exists an $\epsilon>0$ such that $I+\epsilon \Omega_{D}: L \rightarrow L$.

Proof. Since $\Omega_{D}$ is the generator for a finite Markov chain, there is an $\epsilon>0$ so that $\left(I+\epsilon \Omega_{D}\right) g \geqslant 0$ for all $g \geqslant 0$. Now fix $u \in D$, let $g$ be a nonnegative function depending only on coordinates $\eta(x)$ for $x \in D \backslash\{u\}$, and put $h(\eta)=$ $[\eta(u)-\rho] g(\eta)$. In what follows, the variables $x$ and $y$ range over $D \backslash\{u\}$ and $z$ ranges over $S \backslash D$. Then 


$$
\begin{aligned}
\Omega_{D} h(\eta)= & \sum_{x, y} \eta(x)[1-\eta(y)] p(x, y)[\eta(u)-\rho]\left[g\left(\eta_{x y}\right)-g(\eta)\right] \\
& -\eta(u) \sum_{y}[1-\eta(y)] p(u, y)\left[\rho g\left(\eta_{y}\right)+(1-\rho) g(\eta)\right] \\
& +[1-\eta(u)] \sum_{x} \eta(x) p(x, u)\left[(1-\rho) g\left(\eta_{x}\right)+\rho g(\eta)\right] \\
& +g(\eta)\left\{[1-\eta(u)] \sum_{z} \alpha(z) p(z, u)-\eta(u) \sum_{z} p(u, z)[1-\alpha(z)]\right\} \\
= & {[\eta(u)-\rho] \sum_{x, y} p(x, y)\left\{\eta(x)[1-\eta(y)] g\left(\eta_{x y}\right)+\eta(x) \eta(y) g(\eta)\right.} \\
& -[\eta(u)-\rho] g(\eta) \sum_{x, y} p(x, y) \\
& +\eta(u) \sum_{y} p(u, y)\left\{(1-\rho) \eta(y) g(\eta)-\rho[1-\eta(y)] g\left(\eta_{y}\right)\right\} \\
& +[1-\eta(u)] \sum_{x} p(x, u)\left\{\eta(x)(1-\rho) g\left(\eta_{x}\right)-\rho[1-\eta(x)] g(\eta)\right\} \\
& +g(\eta)\left\{[1-\eta(u)]\left[\sum_{z} \alpha(z) p(z, u)+\rho \sum_{x} p(x, u)\right]\right. \\
& \left.-\eta(u)\left[\sum_{z} p(u, z)[1-\alpha(z)]+(1-\rho) \sum_{y} p(u, y)\right]\right\}
\end{aligned}
$$

For $y \in D \backslash\{u\}$, let $g_{0}^{y}(\eta)$ and $g_{1}^{y}(\eta)$ be the functions depending only on the coordinates in $D \backslash\{u, y\}$ which satisfy $g(\eta)=\eta(y) g_{1}^{y}(\eta)+[1-\eta(y)] g_{0}^{y}(\eta)$. Then

$$
\begin{aligned}
& \Omega_{D} h(\eta)+\left[\sum_{x, y} p(x, y)+1-p(u, u)\right] h(\eta) \\
& =[\eta(u)-\rho] \sum_{x, y} p(x, y)\left\{\eta(x)[1-\eta(y)] g\left(\eta_{x y}\right)+\eta(x) \eta(y) g(\eta)+[1-\eta(x)] g(\eta)\right\} \\
& \quad+\eta(u) \sum_{y} p(u, y)[\eta(y)-\rho] g_{1}^{y}(\eta)+[1-\eta(u)] \sum_{x} p(x, u)[\eta(x)-\rho] g_{0}^{x}(\eta) \\
& \quad+g(\eta)\left\{[1-\eta(u)] \sum_{z}[\alpha(z)-\rho] p(z, u)+\eta(u) \sum_{z} p(u, z)[\alpha(z)-\rho]\right\} .
\end{aligned}
$$

So, if the cardinality of $D$ is $n, h+(n+1)^{-1} \Omega_{D} h \in L$, and the proof is complete.

The required stronger form of Corollary 2.6 is then

Corollary 2.12. If $\alpha(x) \geqslant \rho$ on $S \backslash D$ and $\mu>_{s} \nu_{\rho}$, then $\mu S_{D}(t)>_{s} \nu_{\rho}$ for all $t \geqslant 0$.

PROOF. Since the relation $\mu>_{s} \nu_{\rho}$ is preserved by weak convergence, it suffices by Proposition 2.2 to prove the corollary in case $D$ is finite. But, by 
Theorem 2.11 and Proposition 2.1, $S_{D}(t): L \rightarrow L$, and the proof is complete since $\mu>_{s} \nu_{\rho}$ is equivalent to $\int h d \mu \geqslant 0$ for all $h \in L$ by Lemma 2.9.

So far, we have dealt only with inequalities which involve only one $D$ at a time. Throughout the remainder of the paper, however, we will need to compare distributions at time $t$ corresponding to different processes. In doing so, it is important to recall the convention adopted at the beginning of this section regarding the interpretation of $\mu S_{D}(t)$ as a probability measure on $X$.

THEOREM 2.13. Assume that $C \subset D \subset S, \alpha(x) \geqslant \rho$ on $S \backslash D$, and $\alpha(x)=\rho$ on $D \backslash C$. If $\mu>_{s} \nu_{\rho}$, then $\mu S_{C}(t) \leqslant \mu S_{D}(t)$ for all $t>0$.

Proof. By Proposition 2.2, we may assume that $D$ is finite. Consider $f \in M_{C}$. Then

$$
\begin{aligned}
\Omega_{D} f(\eta)-\Omega_{C} f(\eta) & \\
& =\sum_{\substack{x \in C \\
y \in D \backslash C}}\left[f\left(\eta_{x}\right)-f(\eta)\right]\{p(y, x)[1-\eta(x)]-\eta(x) p(x, y)\}[\eta(y)-\rho] .
\end{aligned}
$$

Since the function multiplying $[\eta(y)-\rho]$ is nonnegative, it follows that

$$
\int\left(\Omega_{D} f-\Omega_{C} f\right) d \mu \geqslant 0
$$

whenever $\mu>_{s} \nu_{p}$. Now, by the proof of Theorem 2.3, by Theorem 2.11, and by the fact that $\Omega_{D}$ is the generator of a finite Markov chain, there exists an $\epsilon>0$ so that $\left(I+\epsilon \Omega_{D}\right): M_{D} \rightarrow M_{D},\left(I+\epsilon \Omega_{C}\right): M_{C} \rightarrow M_{C}$,

$$
\left(I+\epsilon \Omega_{D}\right): L \rightarrow L,
$$

and $\left(I+\epsilon \Omega_{D}\right)$ maps nonnegative functions into nonnegative functions. For any $\mu$, let $\tilde{\mu}$ be the probability measure determined by

$$
\int h \tilde{d \mu}=\int\left(h+\epsilon \Omega_{D} h\right) d \mu .
$$

By (2.15), if $\mu>_{s} \nu_{\rho}$, then $\tilde{\mu}>_{s} \nu_{\rho}$. Now let

$$
K=\left\{(f, g) \in M_{C} \times M_{D}: \int f d \mu \leqslant \int g d \mu \text { for all } \mu>_{s} \nu_{\rho}\right\},
$$

and take $(f, g) \in K$ and $\mu>_{s} \nu_{\rho}$. Then

$$
\int\left(g+\epsilon \Omega_{D} g\right) d \mu=\int g d \tilde{\mu} \geqslant \int f \tilde{d \mu}=\int\left(f+\epsilon \Omega_{D} f\right) d \mu \geqslant \int\left(f+\epsilon \Omega_{C} f\right) d \mu,
$$

where the final inequality is a consequence of (2.14). Therefore $\left(f+\epsilon \Omega_{C} f, g+\right.$ $\left.\epsilon \Omega_{D} g\right) \in K$ also. So, the semigroup $\left(S_{C}(t), S_{D}(t)\right)$ maps $K$ into itself. Since $(f, f) \in K$ for $f \in M_{C}$, it follows that 


$$
\int f d\left[\mu S_{C}(t)\right] \leqslant \int f d\left[\mu S_{D}(t)\right]
$$

for $\mu>_{s} \nu_{\rho}$ and $f \in M_{C}$. In order to complete the proof of the theorem, it suffices to prove this inequality for $f \in M_{D}$. For simplicity, assume that $D \backslash C=\{u\}$, a singleton, and if $f \in M_{D}$, write $f(\eta)=\eta(u) f_{1}(\eta)+[1-\eta(u)] f_{0}(\eta)$ where $f_{0}$, $f_{1} \in M_{C}$ and $f_{0} \leqslant f_{1}$. Then

$$
\int f d\left[\mu S_{C}(t)\right]=\int\left[\rho f_{1}+(1-\rho) f_{0}\right] d\left[\mu S_{C}(t)\right]
$$

since $\alpha(u)=\rho$ and by our convention, the random variable $\eta(u)$ is independent of $\{\eta(x), x \in C\}$ relative to the measure $\mu S_{C}(t)$. On the other hand,

$$
\begin{aligned}
\int f d\left[\mu S_{D}(t)\right] & =\int \eta(u)\left[f_{1}-f_{0}\right] d\left[\mu S_{D}(t)\right]+\int f_{0} d\left[\mu S_{D}(t)\right] \\
& \geqslant \rho \int\left[f_{1}-f_{0}\right] d\left[\mu S_{D}(t)\right]+\int f_{0} d\left[\mu S_{D}(t)\right]
\end{aligned}
$$

since $\mu S_{D}(t)>_{s} \nu_{\rho}$ by Corollary 2.12. The result then follows from the fact that $\rho f_{1}+(1-\rho) f_{0} \in M_{C}$.

Corollary 2.16. Assume that $C \subset D \subset S, \alpha(x) \geqslant \rho$ on $S \backslash D$, and $\alpha(x)=$ $\rho$ on $D \backslash C$. If $\nu>_{s} \nu_{\rho}$ and $\nu \geqslant \mu$, then $\mu S_{C}(t) \leqslant \nu S_{D}(t)$ for all $t \geqslant 0$.

3. The finite system. Throughout this section, we will consider the processes described at the beginning of $\S 2$ in the case that $p(x, y)$ is given by (1.1) with $1 / 2<p \leqslant 1$ and

$$
\alpha(x)= \begin{cases}\lambda & \text { if } x \leqslant 0 \\ \rho & \text { if } x>0\end{cases}
$$

Let $D_{n}=\{1, \ldots, n\}, \Omega_{n}=\Omega_{D_{n}}$, and $S_{n}(t)=S_{D_{n}}(t)$. This process is a finite state Markov chain, and is irreducible provided that

$$
p \lambda+q \rho>0 \text { and } p(1-\rho)+q(1-\lambda)>0 .
$$

It is easy to see what the stationary distributions of the chain are if (3.1) fails. If one of the expressions in (3.1) is positive and the other is zero, then the unique stationary distribution is the pointmass on $\eta \equiv 0$ or on $\eta \equiv 1$. If both expressions are zero, then $p=1, \lambda=0$ and $\rho=1$, in which case there are exactly $n+1$ extreme stationary distributions. Therefore throughout this section we will assume that (3.1) holds, and will study the (unique) stationary distribution $\mu_{n}$ of the chain. Our eventual goal is to determine properties of the measure $\lim _{n \rightarrow \infty} \mu_{n}$ on $X_{+}=\{0,1\}^{Z}$, and in particular its dependence on $\lambda$ and $\rho$, since this will be the key to the analysis of the $\lambda$-process in the next section.

The first observation, obtained from the relation $\int \Omega_{n} f d \mu_{n}=0$ for $f(\eta)=$ $\eta(u)$, is that, for $1 \leqslant u \leqslant n-1$, 


$$
\begin{aligned}
p \mu_{n}\{\eta: \eta(u) & =1, \eta(u+1)=0\}-q \mu_{n}\{\eta: \eta(u)=0, \eta(u+1)=1\} \\
& =p \lambda \mu_{n}\{\eta: \eta(1)=0\}-q(1-\lambda) \mu_{n}\{\eta: \eta(1)=1\} \\
& =p(1-\rho) \mu_{n}\{\eta: \eta(n)=1\}-q \rho \mu_{n}\{\eta: \eta(n)=0\}
\end{aligned}
$$

Let $c_{n}$ be the common value of these expressions. The key result which permits the study of $\left\{\mu_{n}, n \geqslant 1\right\}$ is the following recursion relation.

Theorem 3.2. Assume that (3.1) holds, and take $\eta \in\{0,1\}^{D_{n}}$.

(a) If $\eta(u)=1$ and $\eta(u+1)=0$ for some $1 \leqslant u \leqslant n-1$, then

$$
\begin{aligned}
& p \mu_{n}\{\eta\}-q \mu_{n}\left\{\eta_{u, u+1}\right\}=c_{n} \mu_{n-1}\{\zeta: \zeta(x)=\eta(x) \text { for } 1 \leqslant x<u \\
& \text { and } \zeta(x)=\eta(x+1) \text { for } u<x \leqslant n-1\} .
\end{aligned}
$$

(b) If $\eta(1)=0$, then

$$
p \lambda \mu_{n}\{\eta\}-q(1-\lambda) \mu_{n}\left\{\eta_{1}\right\}=c_{n} \mu_{n-1}\{\zeta: \zeta(x)=\eta(x+1) \text { for } 1 \leqslant x \leqslant n-1\} \text {. }
$$

(c) If $\eta(n)=0$, then

$$
p(1-\rho) \mu_{n}\left\{\eta_{n}\right\}-q \rho \mu_{n}\{\eta\}=c_{n} \mu_{n-1}\{\zeta: \zeta(x)=\eta(x) \text { for } 1 \leqslant x \leqslant n-1\} \text {. }
$$

REMARK. The proof of this theorem is considerably simpler when $p=1$, so it is suggested that the interested reader carry it out in that case first.

Proof. Since $\mu_{n}$ is continuous in $p, \rho$ and $\lambda$ whenever (3.1) is satisfied, it suffices to prove the theorem for a dense set of $p, \rho$ and $\lambda$. Therefore we will assume that $p, \rho$ and $\lambda \in(0,1)$ and that

$$
p^{m} \lambda(1-\rho) \neq q^{m} \rho(1-\lambda)
$$

for all integers $m \geqslant 1$. The proof of the theorem will then be by induction on $n$. A simple computation gives

$$
\mu_{1}\{\eta: \eta(1)=1\}=\frac{p \lambda+q \rho}{1+(\lambda-\rho)(p-q)} \text { and } c_{1}=\frac{p^{2} \lambda(1-\rho)-q^{2}(1-\lambda) \rho}{1+(\lambda-\rho)(p-q)} \text {. }
$$

Therefore (a) is vacuous and (b) and (c) hold if $n=1$. Assume then that (a), (b) and (c) hold for $n=1, \ldots, m-1$. Suppose for now that there is a signed measure $\mu$ on $\{0,1\}^{D_{m}}$ such that for each $\eta \in\{0,1\}^{D_{m}}$, the following holds:

(a') If $\eta(u)=1$ and $\eta(u+1)=0$ for some $1 \leqslant u \leqslant m-1$, then

$$
\begin{aligned}
& p \mu\{\eta\}-q \mu\left\{\eta_{u, u+1}\right\}=\mu_{m-1}\{\zeta: \zeta(x)=\eta(x) \text { for } 1 \leqslant x<u \\
& \text { and } \zeta(x)=\eta(x+1) \text { for } u<x \leqslant m-1\} .
\end{aligned}
$$

(b') If $\eta(1)=0$, then

$$
p \lambda \mu\{\eta\}-q(1-\lambda) \mu\left\{\eta_{1}\right\}=\mu_{m-1}\{\zeta: \zeta(x)=\eta(x+1) \text { for } 1 \leqslant x \leqslant m-1\} \text {. }
$$

(c') If $\eta(m)=0$, then 


$$
p(1-\rho) \mu\left\{\eta_{m}\right\}-q \rho \mu\{\eta\}=\mu_{m-1}\{\zeta: \zeta(x)=\eta(x) \text { for } 1 \leqslant x \leqslant m-1\} .
$$

We will show that $\mu$ is a constant multiple of $\mu_{m}$. In order to do so, let $f(\eta)=1$ if $\eta=\zeta$ and $f(\eta)=0$ if $\eta \neq \zeta$ where $\zeta$ is any fixed configuration in $\{0,1\}^{D_{m}}$.

Computing $\Omega_{m} f$ for this $f$ yields

$$
\begin{aligned}
\Omega_{m} f\left(\zeta_{1}\right) & =\zeta(1) p \lambda+[1-\zeta(1)] q(1-\lambda), \\
\Omega_{m} f\left(\zeta_{m}\right) & =[1-\zeta(m)] p(1-\rho)+\zeta(m) q \rho, \\
\Omega_{m} f\left(\zeta_{u, u+1}\right) & =q \zeta(u)[1-\zeta(u+1)]+p \zeta(u+1)[1-\zeta(u)],
\end{aligned}
$$

if $\zeta(u) \neq \zeta(u+1)$,

$$
\begin{aligned}
\Omega_{m} f(\zeta)= & -p \sum_{x=1}^{m-1} \zeta(x)[1-\zeta(x+1)]-q \sum_{x=1}^{m-1} \zeta(x+1)[1-\zeta(x)] \\
& -p \lambda[1-\zeta(1)]-q(1-\lambda) \zeta(1)-p(1-\rho) \zeta(m)-q \rho[1-\zeta(m)],
\end{aligned}
$$

and $\Omega_{m} f(\eta)=0$ for all other $\eta$. Therefore

$\int \Omega_{m} f d \mu=\zeta(1)\left[p \lambda \mu\left\{\zeta_{1}\right\}-q(1-\lambda) \mu\{\zeta\}\right]+[1-\zeta(1)]\left[q(1-\lambda) \mu\left\{\zeta_{1}\right\}-p \lambda \mu\{\zeta\}\right]$

$+\zeta(m)\left[q \rho \mu\left\{\zeta_{m}\right\}-p(1-\rho) \mu\{\zeta\}\right]$

$+[1-\zeta(m)]\left[p(1-\rho) \mu\left\{\zeta_{m}\right\}-q \rho \mu\{\zeta\}\right]$

$+\sum_{u=1}^{m-1} \zeta(u)[1-\zeta(u+1)]\left[q \mu\left\{\zeta_{u, u+1}\right\}-p \mu\{\zeta\}\right]$

$+\sum_{u=1}^{m-1} \zeta(u+1)[1-\zeta(u)]\left[p \mu\left\{\zeta_{u, u+1}\right\}-q \mu\{\zeta\}\right]$.

Using $\left(a^{\prime}\right),\left(b^{\prime}\right)$ and $\left(c^{\prime}\right)$, this becomes

$$
\begin{aligned}
\int \Omega_{m} f d \mu= & {[2 \zeta(1)-1] \mu_{m-1}\{\gamma: \gamma(x)=\zeta(x+1) \text { for } 1 \leqslant x \leqslant m-1\} } \\
& +[1-2 \zeta(m)] \mu_{m-1}\{\gamma: \gamma(x)=\zeta(x) \text { for } 1 \leqslant x \leqslant m-1\} \\
& +\sum_{u=1}^{m-1}[\zeta(u+1)-\zeta(u)] \mu_{m-1}\{\gamma: \gamma(x)=\zeta(x) \text { for } 1 \leqslant x<u \text { and } \\
& \gamma(x)=\zeta(x+1) \text { for } u<x \leqslant m-1\} .
\end{aligned}
$$

But then $\int \Omega_{m} f d \mu=0$ since $[\zeta(u+1)-\zeta(u)] \mu_{m-1}\{\gamma: \gamma(x)=\zeta(x)$ for $1 \leqslant x<$ $u$ and $\gamma(x)=\zeta(x+1)$ for $u<x \leqslant m-1\}=[2 \zeta(u+1)-1] \mu_{m-1}\{\gamma: \gamma(x)=$ $\zeta(x)$ for $1 \leqslant x \leqslant u$ and $\gamma(x)=\zeta(x+1)$ for $u<x \leqslant m-1\}-[2 \zeta(u)-1] \mu_{m-1}$ $\{\gamma: \gamma(x)=\zeta(x)$ for $1 \leqslant x<u$ and $\gamma(x)=\zeta(x+1)$ for $u \leqslant x \leqslant m-1\}$. Therefore 
$\mu$ is a constant multiple of $\mu_{m}$. In fact, summing (a') appropriately yields

$$
p \mu\{\eta: \eta(u)=1, \eta(u+1)=0\}-q \mu \cdot\{\eta: \eta(u)=0, \eta(u+1)=1\}=1,
$$

so $c_{m} \neq 0$ and

$$
\mu=c_{m}^{-1} \mu_{m},
$$

so that (a), (b) and (c) follow from $\left(a^{\prime}\right),\left(b^{\prime}\right)$ and $\left(c^{\prime}\right)$. Therefore, in order to complete the proof, it suffices to prove that there exists a signed measure $\mu$ on $\{0,1\}^{D_{m}}$ which satisfies (a'), $\left(b^{\prime}\right)$ and $\left(c^{\prime}\right)$ for all $\eta \in\{0,1\}^{D_{m}}$. If $\gamma \equiv 1$, define $\mu\{\gamma\}$ by

$$
\begin{aligned}
& {\left[p^{m+1} \lambda(1-\rho)-q^{m+1} \rho(1-\lambda)\right] \mu\{\gamma\}} \\
& =\left[\lambda p^{m}+\rho q^{m}\right] \mu_{m-1}\{\eta: \eta(x)=1 \text { for all } x\} \\
& \quad+\rho \lambda \sum_{y=1}^{m-1} p^{y} q^{m-y} \mu_{m-1}\{\eta: \eta(x)=1 \text { for all } x \neq y\},
\end{aligned}
$$

which is possible by (3.3). Next we will show by induction on $k$ that $\mu\{\eta\}$ can be defined for $\eta$ such that $\Sigma_{x=1}^{m} \eta(x)=m-k$ in such a way that $\left(\mathrm{a}^{\prime}\right),\left(\mathrm{b}^{\prime}\right)$ and $\left(c^{\prime}\right)$ are satisfied for those $\eta$. That this is possible for $k=1$ follows from the above choice of $\mu\{\gamma\}$, as can easily be checked. So, assume that $\mu\{\eta\}$ has been defined for $\eta$ such that $\Sigma_{x=1}^{m} \eta(x)>m-k$ and that $\left(\mathrm{a}^{\prime}\right),\left(\mathrm{b}^{\prime}\right)$ and $\left(\mathrm{c}^{\prime}\right)$ are satisfied for such $\eta$, where $k \geqslant 2$. Use $\left(\mathrm{b}^{\prime}\right)$ and $\left(\mathrm{c}^{\prime}\right)$ to define $\mu\{\eta\}$ for $\eta$ such that $\sum_{x=1}^{m} \eta(x)=m-k$ and $\eta(1)=0$ or $\eta(m)=0$. In order to show that this is well defined if $\eta(1)=0$ and $\eta(m)=0$, it suffices to show that, for such an $\eta$,

$$
\begin{aligned}
& q^{2} \rho(1-\lambda) \mu\left\{\eta_{1}\right\}+q \rho \mu_{m-1}\{\zeta: \zeta(x)=\eta(x+1) \text { for } 1 \leqslant x \leqslant m-1\} \\
& \quad=p^{2} \lambda(1-\rho) \mu\left\{\eta_{m}\right\}-p \lambda \mu_{m-1}\{\zeta: \zeta(x)=\eta(x) \text { for } 1 \leqslant x \leqslant m-1\}
\end{aligned}
$$

By the induction hypothesis, $\left(b^{\prime}\right)$ can be applied to $\eta_{m}$ and $\left(c^{\prime}\right)$ to $\eta_{1}$ to show that (3.6) is equivalent to

$$
\begin{aligned}
& q \rho \mu_{m-1}\{\zeta: \zeta(x)=\eta(x+1) \text { for } 1 \leqslant x \leqslant m-1\} \\
& \quad+p \lambda \mu_{m-1}\{\zeta: \zeta(x)=\eta(x) \text { for } 1 \leqslant x \leqslant m-1\} \\
& =q(1-\lambda) \mu_{m-1}\left\{\zeta: \zeta(x)=\eta_{1}(x) \text { for } 1 \leqslant x \leqslant m-1\right\} \\
& \quad+p(1-\rho) \mu_{m-1}\left\{\zeta: \zeta(x)=\eta_{m}(x+1) \text { for } 1 \leqslant x \leqslant m-1\right\}
\end{aligned}
$$

which is true since (b) and (c) are satisfied for $n=m-1$. Now (a') can be used to define $\mu\{\eta\}$ for $\eta$ such that $\Sigma_{x=1}^{m} \eta(x)=m-k$ and $\eta(1)=\eta(m)=1$. Again, 
to check that this is well defined, and that (a') then holds for all $\eta$ for which $\sum_{x=1}^{m} \eta(x)=m-k$, it suffices to observe that if $\eta(u)=1, \eta(u+1)=0, \eta(v)=$ $1, \eta(v+1)=0$ and $u<v$, then

$$
\begin{array}{r}
p \mu_{m-1}\{\zeta: \zeta(x)=\eta(x) \text { for } 1 \leqslant x<u \text { and } \zeta(x)=\eta(x+1) \text { for } u<x \leqslant m-1\} \\
+q \mu_{m-1}\left\{\zeta: \zeta(x)=\eta_{u, u+1}(x) \text { for } 1 \leqslant x<v \text { and } \zeta(x)=\eta(x+1)\right. \\
\text { for } v<x \leqslant m-1\} \\
=p \mu_{m-1}\{\zeta: \zeta(x)=\eta(x) \text { for } 1 \leqslant x<v \text { and } \zeta(x)=\eta(x+1) \text { for } v<x \leqslant m-1\} \\
+q \mu_{m-1}\left\{\zeta: \zeta(x)=\eta(x) \text { for } 1 \leqslant x<u \text { and } \zeta(x)=\eta_{v, v+1}(x+1)\right. \\
\text { for } u<x \leqslant m-1\} .
\end{array}
$$

But this is a consequence of the fact that (a) holds for $n=m-1$. This completes the induction on $k$, and therefore the proof of the theorem.

In general, it appears to be difficult to express $\mu_{n}$, or even $c_{n}$, explicitly in closed form. It does follow from (3.4) and (3.5) that $c_{n}$ is a positive multiple of $\left[p^{n+1} \lambda(1-\rho)-q^{n+1} \rho(1-\lambda)\right]$, so that if $\lambda(1-\rho)>0$, for example, then $c_{n}$ is positive for large values of $n$. If $p=1$, it can be shown that

$$
\begin{aligned}
& c_{n}=\frac{\lambda(1-\rho) h_{n-1}(\lambda, 1-\rho)}{h_{n}(\lambda, 1-\rho)} \\
& \text { where } h_{n}(x, y)=\sum_{k=0}^{n-1} \frac{n-k}{n+k}\left(\begin{array}{c}
n+k \\
k
\end{array}\right) \sum_{j=0}^{n-k} x^{k+j} y^{n-j},
\end{aligned}
$$

although this will not be needed for the subsequent analysis. If $\lambda=\rho$, then $\mu_{n}=$ $\nu_{\lambda}$ by Theorem 2.4 , and therefore $c_{n}=(p-q) \lambda(1-\lambda)$ for all $n$.

When comparisons are made which involve $\mu_{n}$ for various values of $n$, we will adopt the same convention as in $\S 2: \mu_{n}$ will be viewed as a measure on $\{0,1\} Z$ via

$$
\begin{gathered}
\mu_{n}\left\{\eta: \eta\left(x_{i}\right)=1, \eta\left(y_{j}\right)=1, \eta\left(z_{k}\right)=1 \text { for } 1 \leqslant i \leqslant l, 1 \leqslant j \leqslant m, 1 \leqslant k \leqslant r\right\} \\
=\lambda^{l} \rho^{r} \mu_{n}\left\{\eta: \eta\left(y_{j}\right)=1 \text { for } 1 \leqslant j \leqslant m\right\} \text { for } x_{i} \leqslant 0<y_{j} \leqslant n<z_{k} .
\end{gathered}
$$

We will write $\mu_{n}(\lambda, \rho)$ and $c_{n}(\lambda, \rho)$ when it is necessary to make explicit the dependence on $\lambda$ and $\rho$.

PROPOSITION 3.7. (a) If $\lambda_{1} \leqslant \lambda_{2}$, then $\mu_{n}\left(\lambda_{1}, \rho\right) \leqslant \mu_{n}\left(\lambda_{2}, \rho\right)$.

(b) If $\rho_{1} \leqslant \rho_{2}$, then $\mu_{n}\left(\lambda, \rho_{1}\right) \leqslant \mu_{n}\left(\lambda, \rho_{2}\right)$.

(c) If $\lambda \leqslant \rho$, then $\mu_{n}(\lambda, \rho) \geqslant \mu_{n+1}(\lambda, \rho)$.

(d) If $\lambda \geqslant \rho$, then $\mu_{n}(\lambda, \rho) \leqslant \mu_{n+1}(\lambda, \rho)$.

Proof. Statements (a) and (b) are obtained by letting $t \rightarrow \infty$ in (a) of Theorem 2.4. For (d), apply Theorem 2.13 to $C=D_{n}$ and $D=D_{n+1}$ to obtain $\nu_{\rho} S_{n}(t) \leqslant \nu_{\rho} S_{n+1}(t)$, and then let $t \rightarrow \infty$. The proof of (c) is similar. 
Corollary 3.8. (a) If $\lambda \leqslant \rho$, then $\nu_{\lambda} \leqslant \mu_{n}(\lambda, \rho) \leqslant \nu_{\rho}$.

(b) If $\lambda \geqslant \rho$, then $\nu_{\rho} \leqslant \mu_{n}(\lambda, \rho) \leqslant \nu_{\lambda}$.

Proof. To prove (a), for example, use (a) and (b) of Proposition 3.7 to obtain

$$
\nu_{\lambda}=\mu_{n}(\lambda, \lambda) \leqslant \mu_{n}(\lambda, \rho) \leqslant \mu_{n}(\rho, \rho)=\nu_{\rho}
$$

We will need a slightly stronger form of these inequalities.

Proposition 3.9. (a) If $\lambda<\rho$, then there exists $a \beta<\rho$ such that $\mu_{n}(\lambda, \rho) \leqslant \nu_{\beta}$ on $\{0,1\}^{D_{n}}$.

(b) If $\lambda>\rho$, then there exists $a \beta>\rho$ such that $\mu_{n}(\lambda, \rho) \geqslant v_{\beta}$ on $\{0,1\}^{D_{n}}$.

Proof. The two parts are similar, so assume that $\lambda<\rho$. Since $M_{D_{n}}$ is finite dimensional and $\int f d v_{\beta}$ is continuous in $\beta$ for $f \in M_{D_{n}}$, it suffices to prove that $\int f d \mu_{n}(\lambda, \rho)<\int f d \nu_{\rho}$ for all nonconstant $f \in M_{D_{n}}$. So suppose that $f \in M_{D_{n}}$ and $\int f d \mu_{n}(\lambda, \rho)=\int f d \nu_{\rho}$. Then

$$
\int S_{n}(t) f d \nu_{\rho} \geqslant \int S_{n}(t) f d \mu_{n}(\lambda, \rho)=\int f d \mu_{n}(\lambda, \rho)
$$

where the equality is a consequence of the invariance of $\mu_{n}$ with respect to $S_{n}(t)$, and the inequality follows from Corollary 3.8 and Theorem 2.3. By Theorem 2.4, $\int S_{n}(t) f d \nu_{\rho} \leqslant \int f d \nu_{\rho}$. Putting these inequalities together leads to $\int S_{n}(t) f d \nu_{\rho}=$ $\int f d v_{\rho}$, and therefore to $\int \Omega_{n} S_{n}(t) f d \nu_{\rho}=0$ for all $t \geqslant 0$. But, for any $g \in M_{D_{n}}$,

$$
\int \Omega_{n} g d \nu_{\rho}=(\rho-\lambda) \int[\eta(1)-p]\left[g\left(\eta_{1}\right)-g(\eta)\right] d \nu_{\rho}
$$

and since the integrand is nonpositive, it follows that $S_{n}(t) f\left(\eta_{1}\right)=S_{n}(t) f(\eta)$ for all $\eta$ and all $t \geqslant 0$ if $\rho<1$. But this implies that $f$ is constant. The case $\rho=1$ is easier, and left to the reader. (Recall that $p<1$ by (3.1) if $\rho=1$.)

THEOREM 3.10. (a) $\mu(\lambda, \rho)=\lim _{n \rightarrow \infty} \mu_{n}(\lambda, \rho)$ exists in the sense of weak convergence of measures on $X$.

(b) $c(\lambda, \rho)=\lim _{n \rightarrow \infty} c_{n}(\lambda, \rho)$ exists, $c(\lambda, \rho) \leqslant(p-q) \rho(1-\rho)$ if $\lambda \leqslant \rho$, and $c(\lambda, \rho) \geqslant(p-q) \rho(1-\rho)$ if $\lambda \geqslant \rho$.

(c) If $x_{i}<u$ and $u+1<y_{j}$ for $1 \leqslant i \leqslant k$ and $1 \leqslant j \leqslant l$, then

$$
\begin{gathered}
p \mu(\lambda, \rho)\left\{\eta: \eta\left(x_{i}\right)=1, \eta(u)=1, \eta(u+1)=0, \eta\left(y_{j}\right)=1\right. \\
\qquad \text { for } 1 \leqslant i \leqslant k \text { and } 1 \leqslant j \leqslant l\} \\
-q \mu(\lambda, \rho)\left\{\eta: \eta\left(x_{i}\right)=1, \eta(u)=0, \eta(u+1)=1, \eta\left(y_{j}\right)=1\right. \\
\text { for } 1 \leqslant i \leqslant k \text { and } 1 \leqslant j \leqslant l\} \\
=c(\lambda, \rho) \mu(\lambda, \rho)\left\{\eta: \eta\left(x_{i}\right)=1, \eta\left(y_{j}-1\right)=1 \text { for } 1 \leqslant i \leqslant k \text { and } 1 \leqslant j \leqslant l\right\} .
\end{gathered}
$$


(d) If $x_{i}>1$ for $1 \leqslant i \leqslant k$, then

$$
\begin{gathered}
p \lambda \mu(\lambda, \rho)\left\{\eta: \eta(1)=0, \eta\left(x_{i}\right)=1 \text { for } 1 \leqslant i \leqslant k\right\} \\
-q(1-\lambda) \mu(\lambda, \rho)\left\{\eta: \eta(1)=1, \eta\left(x_{i}\right)=1 \text { for } 1 \leqslant i \leqslant k\right\} \\
=c(\lambda, \rho) \mu(\lambda, \rho)\left\{\eta: \eta\left(x_{i}-1\right)=1 \text { for } 1 \leqslant i \leqslant k\right\} .
\end{gathered}
$$

(e) The relations in (c) and (d) determine $\mu(\lambda, \rho)$ uniquely, so in particular, $\mu(\lambda, \rho)$ depends on $\rho$ only through the value of $c(\lambda, \rho)$.

Proof. If $f \in M$, then $\lim _{n \rightarrow \infty} \int f d \mu_{n}$ exists by (c) and (d) of Proposition 3.7. Therefore (a) follows from the compactness of the set of probability measures on $X$ and the fact that $M$ is a determining class of functions. Since $c_{n}(\lambda, \rho)$ $=p \mu_{n}(\lambda, \rho)\{\eta: \eta(u)=1, \eta(u+1)=0\}-q \mu_{n}(\lambda, \rho)\{\eta: \eta(u)=0, \eta(u+1)=1\}$, it follows from (a) that $\lim _{n \rightarrow \infty} c_{n}(\lambda, \rho)$ exists. Summing (c) of Theorem 3.2 appropriately yields

$$
c_{n}(\lambda, \rho)=p(1-\rho) \mu_{n}(\lambda, \rho)\{\eta: \eta(n)=1\}-q \rho \mu_{n}(\lambda, \rho)\{\eta: \eta(n)=0\}
$$

By Corollary 3.8, if $\lambda \leqslant \rho$, then $\mu_{n}(\lambda, \rho)\{\eta: \eta(n)=1\} \leqslant \rho$, from which $c_{n}(\lambda, \rho)$ $\leqslant(p-q) \rho(1-\rho)$ follows. If $\lambda \geqslant \rho$, then the inequalities are reversed, thus completing the proof of (b). Parts (c) and (d) are obtained by summing and letting $n \rightarrow \infty$ in (a) and (b) of Theorem 3.2. For the proof of (e), consider the problem of determining probabilities of the form $\mu(\lambda, \rho)\left\{\eta: \eta(1)=\epsilon_{1}, \eta(2)=\epsilon_{2}, \ldots\right.$, $\left.\eta(n)=\epsilon_{n}\right\}$ for all choices of $\epsilon_{i}=0$ or 1 . This can be done inductively on $n$ in the following way. For $n=1$, note that (d) determines $\mu(\lambda, \rho)\{\eta: \eta(1)=1\}$. For the induction step, use (c) and (d) to express all the required probabilities in terms of $\mu(\lambda, \rho)\{\eta: \eta(i)=1$ for $1 \leqslant i \leqslant n\}$ if $\lambda>0$, and in terms of $\mu(\lambda, \rho)\{\eta$ : $\eta(i)=0$ for $1 \leqslant i \leqslant n\}$ if $\lambda=0$. The details will be left to the reader.

In order to study the tail behavior of $\mu(\lambda, \rho)$, let $\mu^{n}(\lambda, \rho)$ be the measure which is obtained by shifting $\mu(\lambda, \rho)$ in the following way.

$\mu^{n}(\lambda, \rho)\left\{\eta: \eta\left(x_{i}\right)=1\right.$ for $\left.1 \leqslant i \leqslant k\right\}=\mu(\lambda, \rho)\left\{\eta: \eta\left(x_{i}+n\right)=1\right.$ for $\left.1 \leqslant i \leqslant k\right\}$.

LEMMA 3.11. (a) $\tilde{\mu}(\lambda, \rho)=\lim _{n \rightarrow \infty} \mu^{n}(\lambda, \rho)$ exists.

(b) $\tilde{\mu}(\lambda, \rho)$ is translation invariant.

(c) If $x_{i}<u$ and $u+1<y_{j}$ for $1 \leqslant i \leqslant k$ and $1 \leqslant j \leqslant l$, then

$$
\begin{gathered}
\tilde{p \mu}(\lambda, \rho)\left\{\eta: \eta\left(x_{i}\right)=1, \eta(u)=1, \eta(u+1)=0, \eta\left(y_{j}\right)=1 \text { for } 1<i<k, 1<j<l\right\} \\
-q \tilde{\mu}(\lambda, \rho)\left\{\eta: \eta\left(x_{i}\right)=1, \eta(u)=0, \eta(u+1)=1, \eta\left(y_{j}\right)=1\right. \\
\quad \text { for } 1 \leqslant i<k, 1<j<l\} \\
=c(\lambda, \rho) \tilde{\mu}(\lambda, \rho)\left\{\eta: \eta\left(x_{i}=1, \eta\left(y_{j}-1\right)=1 \text { for } 1<i<k, 1<j<l\right\} .\right.
\end{gathered}
$$


Proof. It follows from an argument similar to that used to prove (c) and (d) of Proposition 3.7 that $\mu^{n}(\lambda, \rho) \leqslant \mu^{n+1}(\lambda, \rho)$ if $\lambda<\rho$ and $\mu^{n}(\lambda, \rho) \geqslant$ $\mu^{n+1}(\lambda, \rho)$ if $\lambda \geqslant \rho$, and (a) is a consequence of this monotonicity. Part (b) is immediate, while part (c) is obtained by using (c) of Theorem 3.10 and then letting $n \rightarrow \infty$.

LEMMA 3.12. $\tilde{\mu}(\lambda, \rho)$ is exchangeable.

Proof. Let $f_{m}(\zeta)=\tilde{\mu}(\lambda, \rho)\{\eta: \eta(x)=\zeta(x)$ for $1 \leqslant x \leqslant m\}$ for $\zeta \in X$. We must show that $f_{m}(\zeta)$ is only a function of $m$ and $k=\Sigma_{x=1}^{m} \zeta(x)$. The proof is by induction on $m$ and then, for each $m$, by induction on $k$. The statement is immediate if $m=1$ or $k=0$ since $\tilde{\mu}(\lambda, \rho)$ is translation invariant. So consider $l \leqslant n$ and assume that $f_{m}(\zeta)$ depends only on $m$ and $k=\Sigma_{x=1}^{m} \zeta(x)$ for $m<n$ and $k \leqslant m$, and for $m=n$ and $k<l$. Put $N=l(n-l)$ and let $\zeta_{0}$ and $\zeta_{N}$ be configurations with $\zeta_{0}(1)=\cdots=\zeta_{0}(l)=1, \zeta_{0}(l+1)=\cdots=\zeta_{0}(n)=0$, $\zeta_{N}(1)=\cdots=\zeta_{N}(n-l)=0$, and $\zeta_{N}(n-l+1)=\cdots=\zeta_{N}(n)=1$. Let $\zeta_{1}$, $\ldots, \zeta_{N-1}$ be configurations such that, for $0 \leqslant i \leqslant N-1, \zeta_{i+1}$ is obtained from $\zeta_{i}$ by interchanging two coordinates $u$ and $u+1$ for which $\zeta_{i}(u)=1$ and $\zeta_{i}(u+1)=0$. By (c) of Lemma 3.11 and the induction hypothesis, $p f_{n}\left(\zeta_{i}\right)-$ $q f_{n}\left(\zeta_{i+1}\right)$ is independent of $i$ and $f_{n}\left(\zeta_{0}\right)=f_{n}\left(\zeta_{N}\right)$. Therefore $f_{n}\left(\zeta_{i}\right)=f_{n}\left(\zeta_{0}\right)$ for all $0 \leqslant i \leqslant N$. This completes the induction step, since for any $\zeta$ such that $\sum_{x=1}^{n} \zeta(x)=l$, the construction of the sequence $\left\{\zeta_{i}\right\}$ can be performed in such a way that $\zeta=\zeta_{i}$ for some $i$.

THEOREM 3.13. If $\rho \geqslant 1 / 2$ and $\lambda+\rho>1$, then $\tilde{\mu}(\lambda, \rho)=\nu_{\rho}$ and therefore $c(\lambda, \rho)=(p-q) \rho(1-\rho)$.

Proof. Since $\tilde{\mu}$ is exchangeable, de Finetti's theorem guarantees the existence of a probability measure $\sigma$ on $[0,1]$ such that $\tilde{\mu}=\int_{0}^{1} v_{\beta} d \sigma(\beta)$. By property (c) of Lemma 3.11,

$$
(p-q) \int_{0}^{1}(1-\beta) \beta^{k+1} d \sigma(\beta)=c(\lambda, \rho) \int_{0}^{1} \beta^{k} d \sigma(\beta) .
$$

Therefore there is some $\beta \in[0,1]$ so that $c(\lambda, \rho)=(p-q) \beta(1-\beta)$, and some $\tau \in[0,1]$ so that $\tilde{\mu}=\tau v_{\beta}+(1-\tau) \nu_{1-\beta}$. By (b) of Theorem $3.10, \beta(1-\beta) \leqslant$ $\rho(1-\rho)$ if $\lambda \leqslant \rho$ and $\beta(1-\beta) \geqslant \rho(1-\rho)$ if $\lambda \geqslant \rho$. By Corollary 3.8, if $\tau>0$, then $\beta$ lies between $\lambda$ and $\rho$, while if $\tau<1$, then $1-\beta$ lies between $\lambda$ and $\rho$. Since $\rho \geqslant 1 / 2$ and $\lambda+\rho>1$, the only possibility is that $\tilde{\mu}=\nu_{\rho}$. The final statement follows from (c) of Lemma 3.11 .

CoRollary 3.14. If $\lambda \leqslant 1 / 2$, then $\lim _{\rho \downarrow 1-\lambda} \mu(\lambda, \rho)=v_{\lambda}$.

Proof. This limit exists by monotonicity, since $\mu\left(\lambda, \rho_{1}\right) \leqslant \mu\left(\lambda, \rho_{2}\right)$ for 
$\rho_{1} \leqslant \rho_{2}$ by (b) of Proposition 3.7. By Theorem 3.13, $\lim _{\rho \downarrow 1-\lambda} c(\lambda, \rho)=$ $(p-q) \lambda(1-\lambda)$ for $\lambda \leqslant 1 / 2$. Therefore the limit is $\nu_{\lambda}$ by (e) of Theorem 3.10.

THEOREM 3.15. If $\lambda \leqslant 1 / 2$ and $\lambda+\rho \leqslant 1$, then $\tilde{\mu}(\lambda, \rho)=\nu_{\lambda}$ and therefore $c(\lambda, \rho)=(p-q) \lambda(1-\lambda)$.

PROOF. By the previous corollary and the monotonicity of $\mu(\lambda, \rho)$ in $\rho$, $\mu(\lambda, \rho) \leqslant v_{\lambda}$. Therefore $\tilde{\mu}(\lambda, \rho) \leqslant v_{\lambda}$. By the argument used in the proof of Theorem 3.13, $\tilde{\mu}(\lambda, \rho)=\nu_{\beta}$ for some $\beta \leqslant \lambda$. Suppose $\beta<\lambda$. Then $c(\lambda, \rho)=$ $(p-q) \beta(1-\beta)=c(\lambda, 1-\beta)$ by Theorem 3.13, so $\mu(\lambda, \rho)=\mu(\lambda, 1-\beta)$ by (e) of Theorem 3.10. But this is impossible since $\tilde{\mu}(\lambda, 1-\beta)=\nu_{1-\beta}$ by Theorem 3.13. Therefore $\beta=\lambda$.

THEOREM 3.16. If $\lambda \geqslant 1 / 2$ and $\rho \leqslant 1 / 2$, then $\tilde{\mu}(\lambda, \rho)=\nu_{1 / 2}$ and therefore $c(\lambda, \rho)=1 / 4(p-q)$.

Proof. Since $\mu(\lambda, \rho) \leqslant \mu(\lambda, 1 / 2)$ by (b) of Proposition 3.7, it follows from Theorem 3.13 that $\tilde{\mu}(\lambda, \rho) \leqslant \widetilde{\mu}(\lambda, 1 / 2)=\nu_{1 / 2}$. Therefore, by the argument used in the proof of Theorem 3.13, $\tilde{\mu}(\lambda, \rho)=v_{\beta}$ for some $\beta \leqslant 1 / 2$. Suppose $\beta<1 / 2$. Then $c(\lambda, \rho)=(p-q) \beta(1-\beta)=c(\lambda, 1-\beta)$ by Theorem 3.13, so $\mu(\lambda, \rho)=$ $\mu(\lambda, 1-\beta)$ by (e) of Theorem 3.10. But this is impossible since $\tilde{\mu}(\lambda, 1-\beta)=$ $\nu_{1-\beta}$ by Theorem 3.13 , so it follows that $\beta=1 / 2$.

Finally, we will summarize some of the previous results in the following way.

Corollary 3.17. (a) If $\lambda \geqslant 1 / 2$ and $\rho \leqslant 1 / 2$, then $\mu(\lambda, \rho)=\mu(\lambda, 1 / 2)$.

(b) If $\lambda \leqslant 1 / 2$ and $\lambda+\rho \leqslant 1$, then $\mu(\lambda, \rho)=\nu_{\lambda}$.

(c) If $\rho \geqslant 1 / 2$ and $\lambda+\rho>1$, then $\mu(\lambda$, $\rho)$ behaves like $\nu_{\rho}$ at $\infty$.

(d) $c(\lambda, \rho)$ is continuous on $[0,1] \times[0,1]$.

(e) $\mu(\lambda, \rho)$ is continuous in the sense of weak convergence on $[0,1] \times$ $[0,1]$.

Proof. (a), (b) and (c) follow from Theorems 3.16, 3.15 and 3.13, together with (e) of Theorem 3.10. Since $c(\lambda, \rho)$ has been computed explicitly, (d) is immediate. The final statement follows from this and (e) of Theorem 3.10.

4. The one-sided system. This section is devoted to the ergodic theory of the $\lambda$-process, which is obtained from the processes described at the beginning of $\S 2$ by taking $S=Z, p(x, x+1)=p, p(x, x-1)=q$, where $p+q=1$ and $1 / 2<p \leqslant 1$, and $\alpha(0)=\lambda$. As in the introduction, the generator and semigroup corresponding to this process will be denoted by $\Omega_{\lambda}$ and $S_{\lambda}(t)$ respectively. In order to emphasize the dependence on $\lambda$ and $\rho$ of the semigroups from $\S 3$, we will write $S_{n}(t)=S_{n}(t ; \lambda, \rho)$. In $\S 3, \mu(\lambda, \rho)$ was defined whenever (3.1) is satisfied. In order to define it in the other cases, let $\mu(\lambda, \rho)$ be the pointmass on $\eta \equiv 0$ if $p \lambda+q \rho=0$, and the pointmass on $\eta \equiv 1$ if $p \lambda+q \rho>0$ and $p(1-\rho)$ 
$+q(1-\lambda)=0$. These cases are generally simpler than those in which (3.1) holds. Special arguments which arc at times required for them will usually be omitted.

THEOREM 4.1. For each $\rho \in[0,1], \mu(\lambda, \rho)$ is invariant for the $\lambda$-process.

Proof. By Proposition 2.2, $S_{n}(t ; \lambda, \rho) f \rightarrow S_{\lambda}(t) f$ uniformly on $X_{+}$for all $f$ which depend on finitely many coordinates. Since $\mu_{n}(\lambda, \rho)$ is invariant for $S_{n}(t ; \lambda, \rho)$,

$$
\int S_{n}(t ; \lambda, \rho) f d \mu_{n}(\lambda, \rho)=\int f d \mu_{n}(\lambda, \rho)
$$

Therefore $\int S_{\lambda}(t) f d \mu(\lambda, \rho)=\int f d \mu(\lambda, \rho)$ by (a) of Theorem 3.10, from which the result follows.

LEMMA 4.2. If $\rho \geqslant 1 / 2$ and $\lambda+\rho>1$, then $\nu_{\rho} S_{\lambda}(t) \rightarrow \mu(\lambda, \rho)$ as $t \rightarrow \infty$.

Proof. Assume that $\lambda \geqslant \rho$, since the proof is similar in the other case. Then $\nu_{\rho} \leqslant \mu(\lambda, \rho)$ by Corollary 3.8 , so $\nu_{\rho} S_{\lambda}(t) \leqslant \mu(\lambda, \rho)$ by Theorems 2.3 and 4.1. On the other hand, $\nu_{\rho} S_{\lambda}(t) \leqslant \nu_{\rho} S_{n}(t ; \lambda, \rho)$ by Theorem 2.13, and $\lim _{n \rightarrow \infty} \lim _{t \rightarrow \infty} \nu_{\rho} S_{n}(t ; \lambda, \rho)=\mu(\lambda, \rho)$ by (a) of Theorem 3.10. Therefore every weak limit of $\nu_{\rho} S_{\lambda}(t)$ as $t \rightarrow \infty$ is equal to $\mu(\lambda, \rho)$, and the result follows by compactness.

Lemma 4.3. Assume that $\mu$ is a product measure on $X_{+}$.

(a) If $\lambda \geqslant 1 / 2$ and $\mu\{\eta: \eta(x)=1\} \leqslant 1 / 2$ for all $x \in Z_{+}$, then

$$
\mu S_{\lambda}(t) \rightarrow \mu(\lambda, 1 / 2) \text { as } t \rightarrow \infty \text {. }
$$

(b) If $0<\lambda \leqslant 1 / 2$ and $\mu\{\eta: \eta(x)=1\} \leqslant 1-\lambda$ for all $z \in Z_{+}$, then

$$
\mu S_{\lambda}(t) \rightarrow \nu_{\lambda} \text { as } t \rightarrow \infty
$$

Proof. In order to prove (a), take $C=\{1, \ldots, n\}, D=Z_{+}$and $\rho=0$ in Theorem 2.13 to obtain $v_{0} S_{\lambda}(t) \geqslant v_{0} S_{n}(t ; \lambda, 0)$. By (a) of Theorem 3.10 and (a) of Corollary 3.17 ,

$$
\lim _{n \rightarrow \infty} \lim _{t \rightarrow \infty} \nu_{0} S_{n}(t ; \lambda, 0)=\mu(\lambda, 0)=\mu(\lambda, 1 / 2) .
$$

On the other hand, $\nu_{0} \leqslant \mu(\lambda, 1 / 2)$, so $\nu_{0} S_{\lambda}(t) \leqslant \mu(\lambda, 1 / 2)$ for all $t \geqslant 0$ by Theorems 2.3 and 4.1. Therefore $\lim _{t \rightarrow \infty} \nu_{0} S_{\lambda}(t)=\mu(\lambda, 1 / 2)$. Since $\nu_{0} \leqslant \mu \leqslant \nu_{1 / 2} \leqslant \mu(\lambda, 1 / 2)$ by Corollary 3.8, another application of Theorem 2.3 gives the required result. For (b), the same argument shows that $\lim _{t \rightarrow \infty} \nu_{0} S_{\lambda}(t)=\nu_{\lambda}$. By Lemma 4.2 and Corollary 3.14, $\lim _{\rho \downarrow 1-\lambda} \lim _{t \rightarrow \infty} \nu_{\rho} S_{\lambda}(t)=\nu_{\lambda}$. Since $\nu_{0} \leqslant \mu \leqslant \nu_{\rho}$ for $\rho>1-\lambda$, the result follows from Theroem 2.3.

Lemma 4.4. Assume that $1>\rho \geqslant 1 / 2, \lambda+\rho>1$, and $\lambda \neq \rho$. Then for each $n$ there is $a \beta$ with $\beta<\rho$ if $\lambda<\rho$ and $\beta>\rho$ if $\lambda>\rho$ such that if $\mu$ is a 
product measure on $X_{+}$with $\mu\{\eta: \eta(x)=1\}$ between $\beta$ and $\rho$ for $1 \leqslant x \leqslant n$ and $\mu\{\eta: \eta(x)=1\}=\rho$ for $x>n$, then $\mu S_{\lambda}(t) \rightarrow \mu(\lambda, \rho)$ as $t \rightarrow \infty$.

Proof. Assume for simplicity that $\lambda<\rho$. By Proposition 3.9, given $n$, there exists a $\beta<\rho$ such that $\mu_{n}(\lambda, \rho) \leqslant \nu_{\beta}$ on $\{0,1\}^{D_{n}}$. Let $\mu$ be a product measure on $X_{+}$with $\rho \geqslant \mu\{\eta: \eta(x)=1\} \geqslant \beta$ for $1 \leqslant x \leqslant n$ and $\mu\{\eta: \eta(x)=1\}$ $=\rho$ for $x>n$. Then

$$
\mu(\lambda, \rho) \leqslant \mu_{n}(\lambda, \rho) \leqslant \mu \leqslant \nu_{\rho},
$$

so $\mu S_{\lambda}(t) \rightarrow \mu(\lambda, \rho)$ by Lemma 4.2 and Theorem 2.3 .

Theorem 1.7 is contained in Theorem 4.1 and Corollaries 3.14 and 3.17. We now complete the proof of Theorem 1.8, special cases of which appear in Lemmas 4.3 and 4.4 .

Proof of TheOREM 1.8. The additional arguments needed to generalize from Lemmas 4.3 and 4.4 to Theorem 1.8 are similar in the various cases which occur. Therefore we will carry them out only in the case that $1 / 2<\lambda<\rho<1$. Fix $n$ for the moment, and let $\eta^{1}, \ldots, \eta^{N}$ be the points of $\{0,1\}^{D_{n}}$, where $N=2^{n}$. For $1 \leqslant i \leqslant N$, let $\mu_{i}$ be the product measure on $X_{+}$with $\mu_{i}\{\eta: \eta(x)=1\}=$ $\eta^{i}(x)$ for $1 \leqslant x \leqslant n$ and $\mu_{i}\{\eta: \eta(x)=1\}=\rho$ for $x>n$. Let $\beta$ be as in Lemma 4.4 , and let $\beta_{j}$ be such that $\beta \leqslant \beta_{j} \leqslant \rho$ for $1 \leqslant j \leqslant n$. Then if

$$
\mu=\sum_{i=1}^{N}\left[\prod_{j=1}^{n} \beta_{j}^{n^{i}(j)}\left(1-\beta_{j}\right)^{1-\eta^{i}(j)}\right] \mu_{i}
$$

it follows from Lemma 4.4 that $\mu S_{\lambda}(t) \rightarrow \mu(\lambda, \rho)$. Since this is true for all choices of $\beta_{j}$ 's satisfying the given constraints, it follows that $\mu_{i} S_{\lambda}(t) \rightarrow \mu(\lambda, \rho)$ for each $i$. Therefore $\mu S_{\lambda}(t) \rightarrow \mu(\lambda, \rho)$ whenever $\mu$ is a product measure satisfying $\mu\{\eta: \eta(x)=1\}=\rho$ for $x \geqslant n$. Now suppose $\mu$ is a product measure satisfying $\lim _{x \rightarrow \infty} \mu\{\eta: \eta(x)=1\}=\rho$. Then, for every $\epsilon>0$, there is an $n$ and product measures $\underline{\mu}$ and $\bar{\mu}$ with $\underline{\mu}\{\eta: \eta(x)=1\}=\rho-\epsilon$ and $\bar{\mu}\{\eta: \eta(x)=1\}=\rho+\epsilon$ for $x \geqslant n$ such that $\underline{\mu} \leqslant \mu \leqslant \bar{\mu}$. By Theorem 2.3 and the preceding case, all weak limits of $\mu S_{\lambda}(t)$ lie between $\mu(\lambda, \rho-\epsilon)$ and $\mu(\lambda, \rho+\epsilon)$. Therefore $\mu S_{\lambda}(t) \rightarrow$ $\mu(\lambda, \rho)$ by (e) of Corollary 3.17. One other case which merits additional comments is that in which $\lambda=0$ and $\rho=1$. If $p<1$, then (3.1) holds, so the results of $\S 3$ are available. By Theorem $2.13, v_{1} S_{\lambda}(t) \leqslant v_{1} S_{n}(t ; 0,1)$, so $v_{1} S_{\lambda}(t) \rightarrow v_{0}$ since $\lim _{n \rightarrow \infty} \lim _{t \rightarrow \infty} \nu_{1} S_{n}(t ; 0,1)=\nu_{0}$ by (b) of Corollary 3.17. On the other hand, if $p=1$ and $\Sigma_{x \geqslant 1} \mu\{\eta: \eta(x)=0\}=\infty$, then $\mu$ concentrates on $\left\{\eta \in X_{+}\right.$: $\eta(x)=0$ for infinitely many $x \geqslant 1\}$, and therefore $\mu S_{\lambda}(t) \rightarrow \nu_{0}$ is immediate.

5. The two-sided system. This section is devoted to the analysis of the simple exclusion process on $Z$ with $p(x, x+1)=p$ and $p(x, x-1)=q$, where $p+q=1$ and $p>q$, and in particular to the proof of Theorem 1.3. In order to 
reduce the number of cases that need to be considered, it is convenient to use the following symmetry principle, whose proof follows easily from an examination of the form of the generator $\Omega$. From it, for example, follows that part (b) of Theorem 1.3 implies part (c), and that it suffices to prove part (a) in case $\lambda+$ $\rho \geqslant 1$.

Proposition 5.1. If $\left\{\eta_{t}(x): x \in Z\right\}$ moves according to the simple exclusion process, then so does $\left\{1-\eta_{t}(-x): x \in Z\right\}$ (with the same $p$ ).

Proof of Theorem 1.3. In order to prove part (a), first let $\mu$ be any product measure on $X$ such that

$$
\lim _{x \rightarrow \infty} \mu\{\eta: \eta(x)=1\} \leqslant 1 / 2 .
$$

Let $\nu$ be the product measure on $X$ with $\nu\{\eta: \eta(x)=1\}=1$ for $x \leqslant 0$ and $\nu\{\eta: \eta(x)=1\}=\mu\{\eta: \eta(x)=1\}$ for $x \geqslant 1$. Take $C=Z_{+}$and $D=Z$ in the version of Corollary 2.16 obtained by interchanging the roles of 0 and 1 to conclude that $\mu S(t) \leqslant \nu S_{\lambda}(t)$ with $\lambda=1$ for all $t \geqslant 0$. Therefore if $\mu_{\infty}$ is any weak limit of $\mu S(t)$ as $t \rightarrow \infty$, it follows from (a) of Theorem 1.8 that $\mu_{\infty} \leqslant \mu(1,1 / 2)$. Since condition (5.2) is not changed by shifting $\mu$ by a finite amount, and since $\mu(1,1 / 2)$ behaves like $\nu_{1 / 2}$ at $\infty$, it follows that $\mu_{\infty} \leqslant \nu_{1 / 2}$. By Proposition 5.1, it then follows that if $\mu$ is any product measure on $X$ such that

$$
\lim _{x \rightarrow-\infty} \mu\{\eta: \eta(x)=1\} \geqslant 1 / 2
$$

then all weak limit points $\mu_{\infty}$ of $\mu S(t)$ as $t \rightarrow \infty$ satisfy $\mu_{\infty} \geqslant \nu_{1 / 2}$. Therefore if $\mu$ is a product measure on $X$ which satisfies both (5.2) and (5.3), it follows that $\lim _{t \rightarrow \infty} \mu S(t)$ exists and is $\nu_{1 / 2}$, thus concluding the proof of part (a). For the proof of (b), assume first that

$$
\rho>\max (\lambda, 1-\lambda)
$$

and that $\mu$ is a product measure on $X$ which satisfies

$$
\lambda \leqslant \mu\{\eta: \eta(x)=1\} \leqslant \rho
$$

in addition to (1.4). Take $C=Z_{+}$and $D=Z$ with $\lambda$ in place of $\rho$ in Theorem 2.13 to obtain $\mu S_{\lambda}(t) \leqslant \mu S(t)$ for all $t \geqslant 0$. Therefore if $\mu_{\infty}$ is any weak limit of $\mu S(t)$ as $t \rightarrow \infty, \mu_{\infty} \geqslant \mu(\lambda, \rho)$ by Theorem 1.8. Since $\mu(\lambda, \rho)$ behaves like $\nu_{\rho}$ at $\infty, \mu_{\infty} \geqslant \nu_{\rho}$ is obtained by shifting $\mu$. On the other hand, since $\mu \leqslant \nu_{\rho}$, it follows that $\mu S(t) \leqslant \nu_{\rho}$ by Theorems 2.3 and 2.4. Therefore $\mu_{\infty} \leqslant \nu_{\rho}$, so $\lim _{t \rightarrow \infty} \mu S(t)$ exists and is $\nu_{\rho}$. Now the arguments used in the proof of Theorem 1.8 can be used to eliminate restriction (5.5), and therefore to complete the proof of (b) in case (5.4) holds. Now assume that $1 / 2 \leqslant \rho<\lambda$ and that $\mu$ is a product measure 
on $X$ which satisfies

$$
\rho \leqslant \mu\{\eta: \eta(x)=1\} \leqslant \lambda
$$

in addition to (1.4). Using Theorem 2.13 again gives $\mu S(t) \leqslant \mu S_{\lambda}(t)$ for all $t \geqslant 0$, so that if $\mu_{\infty}$ is any weak limit of $\mu S(t)$ as $t \rightarrow \infty$, it follows that $\mu_{\infty} \leqslant \mu(\lambda, \rho)$ by Theorem 1.8. Since $\mu(\lambda, \rho)$ behaves like $\nu_{\rho}$ at $\infty$, it follows that $\mu_{\infty} \leqslant \nu_{\rho}$. Since $\mu \geqslant \nu_{\rho}$, it again follows that $\mu_{\infty} \geqslant \nu_{\rho}$, and therefore that $\lim _{t \rightarrow \infty} \mu S(t)=\nu_{\rho}$. Restriction (5.6) is then removed as before. The one remaining situation to be considered in part (b) is $\lambda=\rho>1 / 2$. So, suppose that $\mu$ is a product measure on $X$ satisfying (1.4) in this case. Given $0<\epsilon<\rho-1 / 2$, let $\underline{\mu}$ and $\bar{\mu}$ be product measures on $X$ whose densities at $-\infty$ and $+\infty$ exist and are given by $\underline{\lambda}=\lambda-\epsilon$, $\rho=\rho, \bar{\lambda}=\lambda$ and $\bar{\rho}=\rho+\epsilon$, and which satisfy $\underline{\mu} \leqslant \mu \leqslant \bar{\mu}$. Then, by the cases already considered, $\lim _{t \rightarrow \infty} \underline{\mu} S(t)=\nu_{\rho}$ and $\lim _{t \rightarrow \infty} \bar{\mu} S(t)=\nu_{\rho+\epsilon}$; so if $\mu_{\infty}$ is any weak limit of $\mu S(t)$ as $t \rightarrow \infty$, it follows that $\nu_{\rho} \leqslant \mu_{\infty} \leqslant \nu_{\rho+\epsilon}$. Therefore $\lim _{t \rightarrow \infty} \mu S(t)=\nu_{\rho}$.

THEOREM 5.7. If $0 \leqslant \lambda<1 / 2$ and $\lambda+\rho=1$, then there exists a product measure $\mu$ on $X$ which satisfies (1.4) such that both $\nu_{\lambda}$ and $\nu_{\rho}$ are weak limit points of $\mu S(t)$ as $t \rightarrow \infty$.

Proof. Let $\left\{f_{k}: k \geqslant 1\right\}$ be a sequence in $M$ whose linear span is dense in $C(X)$. Whenever $\mu$ appears in the proof, it will be a product measure on $X$ which satisfies $\lambda \leqslant \mu\{\eta: \eta(x)=1\} \leqslant \rho$ and $\mu\{\eta: \eta(x)=1\}$ is nondecreasing in $x$. It suffices to prove that there are sequences $t_{n} \uparrow \infty, \alpha_{n}$, and $x_{n}$ such that $\alpha_{0}=$ $1 / 2<\alpha_{1}, \alpha_{2 n} \downarrow \lambda, \alpha_{2 n+1} \uparrow \rho, x_{n}$ is an integer, $x_{0}=0>x_{1}, x_{2 n} \uparrow \infty$, and $x_{2 n+1} \downarrow-\infty$, and such that, for each $n \geqslant 1$,

$$
\begin{aligned}
& \int S\left(t_{n}\right) f_{k} d \mu<\int f_{k} d \nu_{\lambda}+\frac{1}{n} \text { if } n \text { is even, } \\
& \int S\left(t_{n}\right) f_{k} d \mu>\int f_{k} d \nu_{\rho}-\frac{1}{n} \quad \text { if } n \text { is odd }
\end{aligned}
$$

whenever $1 \leqslant k \leqslant n$ and $\mu$ satisfies

$$
\mu\{\eta: \eta(x)=1\}= \begin{cases}\alpha_{0} & \text { if } x_{1}<x<x_{0}, \\ \alpha_{i} & \text { if } x_{i-1} \leqslant x<x_{i+1}, i \text { odd, } 1 \leqslant i<n, \\ \alpha_{i} & \text { if } x_{i+1}<x \leqslant x_{i-1}, i \text { even, } 1 \leqslant i<n, \\ \alpha_{n} & \text { if } x=x_{n-1} .\end{cases}
$$

The three sequences will be constructed recursively. So, assume that they have been chosen for $n<l$, and assume for specificity that $l$ is even. Let $\mu$ be the measure which satisfies (5.9) for $n=l-1, \mu\{\eta: \eta(x)=1\}=\alpha_{l-1}$ for $x \geqslant x_{l-2}$, and $\mu\{\eta: \eta(x)=1\}=\lambda$ for $x \leqslant x_{l-1}$. Then $\mu S(t) \rightarrow \nu_{\lambda}$ by Theorem 1.3, so there is a $t_{l}>t_{l-1}$ such that (5.8) holds for $n=l$ and $1 \leqslant k \leqslant l$. Since $S\left(t_{l}\right) f_{k} \in$ 
$C(X)$ for each $k, x_{l}$ and $\alpha_{l}$ can be chosen so that (5.8) holds whenever $\mu$ satisfies (5.9) with $n=l$.

ACKNowledgement. I would like to thank the Mathematics Department of Cornell University for its hospitality during the Spring semester of 1974 . During that time, I benefited from many conversations with F. Spitzer and H. Kesten. I am particularly indebted to F. Spitzer for sharing with me his conjecture of the special case of Theorem 1.3 which is mentioned in the abstract, and for suggesting that a version of the $\lambda$-process might be relevant to its proof.

\section{REFERENCES}

1. R. Holley, $A$ class of interactions in an infinite particle system, Advances in Math. 5 (1970), 291-309. MR 42 \#3857.

2. - Pressure and Helmholtz free energy in a dynamic model of a lattice gas, Proc. Sixth Berkeley Sympos. on Math. Statistics and Probability, vol. III, pp. 565-578.

3. T. M. Liggett, $A$ characterization of the invariant measures for an infinite particle system with interactions, Trans. Amer. Math. Soc. 179 (1973), 433-453. MR 48 \#5209.

4. - A characterization of the invariant measures for an infinite particle system with interactions. II, Trans. Amer. Math. Soc. 198 (1974), 201-213.

5. - Convergence to total occupancy in an infinite particle system with interactions, Ann. Probability 2 (1974), 989-998.

6. Existence theorems for infinite particle systems, Trans. Amer. Math. Soc. 165 (1972), 471-481. MR 46 \#8328.

7. P. Meyer, Probability and potentials, Blaisdell, Waltham, Mass., 1966. MR 34 \#5119.

8. F. Spitzer, Interaction of Markov processes, Advances in Math. 5 (1970), 246290. MR $42 \# 3856$.

9. - Recurrent random walk of an infinite particle system, Trans. Amer. Math. Soc. 198 (1974), $191-199$.

DEPARTMENT OF MATHEMATICS, UNIVERSITY OF CALIFORNIA, LOS ANGELES, CALIFORNIA 90024 